\title{
Predicting toxicity caused by high-dose-rate brachytherapy single boost for prostate cancer
}

\author{
Johan Staby Olsén, MD*, Dalia Estefan, MB²*, Antonios Valachis, PhD³, Frida Jakobsson, MD³, Leif Karlsson, PhLic \\ Bengt Johansson, $\mathrm{PhD}^{3}$ \\ *Johan Staby Olsén and Dalia Estefan contributed equally to this work. \\ 'Department of Oncology, General Hospital of Karlstad, Sweden, ${ }^{2}$ School of Medical Sciences, Örebro University, Örebro, Sweden, \\ ${ }^{3}$ Department of Oncology, Faculty of Medicine and Health, Örebro University, Örebro, Sweden, ${ }^{4}$ Department of Medical Physics, \\ Örebro University Hospital, Örebro, Sweden
}

\begin{abstract}
Purpose: Treating localized prostate cancer (PC) with combination radiotherapy consisting of external beam radiotherapy (EBRT) and high-dose-rate brachytherapy (HDR-BT) has been proven to result in better disease outcome than EBRT only. We aimed to evaluate the incidence of toxicities due to combination therapy and identify parameters correlated to acute or late urinary, rectal, and erectile toxicities.

Material and methods: Data on symptoms and tumor/treatment parameters were collected from 359 patients treated between 2008 and 2018 with EBRT (42 Gy in 14 fractions) and HDR-BT (14.5 Gy in one fraction) for localized PC, at the Örebro University Hospital. Urinary, rectal, and erectile symptoms were presented descriptively, and bivariate analyses for correlation between grade $\geq 2$ toxicity and potential predictors were performed. To evaluate prognostic models, multivariable analyses were applied.

Results: Urinary toxicity grade $\geq 2$ was observed in 154 patients $(47 \%$ of patients without pre-existing symptoms grade $\geq 2$ ), of which 15 were grade 3 . Rectal toxicity grade 2 was observed in $22(6 \%)$ patients. Any grade erectile dysfunction was evident in all patients without pre-existing dysfunction $(n=103)$, whereas only 7 recovered completely. In bivariate analyses age was correlated with higher risk of acute urinary toxicity, and irradiated volume was associated with both urinary and rectal toxicities. However, we found no multivariable model of clinical and statistical significance to predict the risk of urinary or rectal toxicities.

Conclusions: In our study cohort, the severity of toxicities was in general mild or moderate and temporary, whereas the incidence of severe toxicity was considerably low. Although we found no predictive models for toxicities, our findings are reassuring that this treatment approach as curative therapy for localized PC is well-tolerated.

J Contemp Brachytherapy 2022; 14, 1: 7-14 DOI: https://doi.org/10.5114/jcb.2022.113545
\end{abstract}

Key words: prostate cancer, toxicity, predictive model, HDR, brachytherapy, boost, hypo-fractionation.

\section{Purpose}

Prostate cancer (PC) is the second most commonly occurring cancer in men, with 1.3 million new cases and 360,000 deaths from PC worldwide in 2018 [1].

For localized PC, the curative treatment strategies available are radical prostatectomy or radiation therapy. Radiation therapy can be delivered by different modalities, such as external beam radiation therapy (EBRT), low-doserate brachytherapy (LDR-BT, permanently implanted radioactive seeds), and high-dose-rate brachytherapy (HDRBT). For low-risk and favorable intermediate-risk PC, brachytherapy as monotherapy is a common treatment approach. Combining EBRT with brachytherapy as a boost is indicated for intermediate- and high-risk PC patients [2].
Apart from considering the clinical characteristics of PC before choosing the most suitable curative treatment approach, current guidelines suggest shared decision-making based on patient's preferences, life expectancy, prior symptoms, and expected treatment-related adverse effects, mainly urinary or rectal toxicity and erectile dysfunction [3-5].

Several studies have been performed to compare disease control rate between different treatment strategies, showing that radiotherapy and surgery have comparable efficacy but different toxicity profiles [3-5]. Studies comparing various radiotherapy treatment modalities collectively imply that combining brachytherapy with EBRT seems to be superior to EBRT alone [6, 7]. In fact,
Address for correspondence: Bengt Johansson, MD, PhD, Department of Oncology, Örebro University Hospital, SE-70185 Örebro, Sweden, « e-mail: bengt.johansson@regionorebrolan.se
Received: 21.01.2021

Accepted: 06.12 .2021

Published: 18.02.2022 
a randomized controlled study analyzed 216 patients with low-, intermediate-, and high-risk PC, and showed a $31 \%$ reduction of recurrence-risk with HDR-BT combined with EBRT compared to treatment with EBRT alone [8]. Despite the potential benefit of HDR-BT and EBRT combination therapy, a decreasing trend in utilization of this treatment approach has been observed in intermediate- and high-risk PC patients $[9,10]$. The proposed reasons for this decline include decreased training in brachytherapy, limited capacities of radiation facilities in delivering brachytherapy, and invasiveness of the treatment $[9,10]$. Furthermore, as some studies have shown, an increased risk of urinary toxicity when using dose escalation with combination therapy and concerns for adverse effects could be another reason for the decreasing trend [10].

To facilitate physicians in predicting toxicity risks and therefore in planning, the most favorable treatment for PC patients with predictive models that estimate the probability of a certain toxicity outcome have been developed [11-15]. Interestingly, most of these models predict outcomes of interest following EBRT, and scarcely any predicting toxicity after treatment with combined HDRBT and EBRT can be found $[16,17]$.

Using a retrospective study design from a prospectively collected database, and including consecutive PC patients treated with combination of EBRT and HDR-BT, we aimed to evaluate the toxicity rates of this treatment approach. Considering the scarce evidence on the predictive role of different parameters, we also aimed to identify treatment-, patient-, and tumor-related risk factors for developing acute and late urinary, rectal and erectile toxicities, and if possible, create predictive models for acute and late toxicities of grade $\geq 2$. Such models will potentially help physicians to be more confident in expected toxicities and increase their use of combination therapy.

\section{Material and methods}

\section{Study cohort}

A retrospective cohort study was conducted, including consecutive patients with histologically confirmed intermediate- and high-risk PC, treated with combination therapy of EBRT and HDR-BT with curative intention and identical total doses in the Department of Oncology at Örebro University Hospital (ÖUH) between 2008 and 2018.

The treatment consisted of EBRT with a total dose of 42 Gy in 14 fractions (3 Gy/fraction 3 times a week), starting 2 weeks after a HDR-BT boost of 14.5 Gy in 1 fraction. Total equal dose with $2 \mathrm{~Gy}$ per fraction $\left(\mathrm{EQD}_{2}\right)$ for this schedule was $101 \mathrm{~Gy}(\alpha / \beta=3)$ referring to $P C$ cells and late reacting tissue, and $75 \mathrm{~Gy}(\alpha / \beta=10)$ for acute reacting tissue. Fiducials were applied during the brachytherapy treatment. EBRT was given with 3 dimensional conformal EBRT from 2008-2013 (3D-CRT), and volumetric modulated arc therapy (VMAT) was applied later on. Clinical target volume (CTV) was defined as prostate capsule seen on computer tomography for EBRT and on ultrasound (US) for HDR-BT. Planning target volume (PTV) was de- fined as CTV +7 mm for EBRT, and PTV = CTV for HDRBT. Risk organs (urethra and rectum) were delineated $5 \mathrm{~mm}$ beyond CTV for HDR-BT. Dose constraints and how HDR-BT is applied are published elsewhere [18].

Patients were derived from a prospectively collected database of 2,517 PC patients treated with radiation therapy with curative intention.

We excluded patients with lymph node metastasis or distant metastatic disease at diagnosis, patients treated with EBRT only or BT only, patients who received combined EBRT and HDR-BT with other doses than those mentioned above, and patients who did not complete the planned course of radiotherapy.

Using the above-mentioned inclusion and exclusion criteria, 359 patients were eligible and included in the study cohort.

\section{Data collection}

Data extracted from the database included tumor characteristics, treatment-related parameters, and toxicity at baseline and during follow-up. Dosimetric data retrieved from brachytherapy dose planning system were included in the database. However, no dosimetric parameters from EBRT were included.

\section{Outcomes of interest, definitions, and follow-up}

Patients were followed-up according to the institution's clinical practice, with a clinical visit at the end of radiotherapy, telephone follow-up with an oncology nurse 3 weeks after the end of radiotherapy, and a standardized questionnaire sent by mail every 6 months after radiotherapy during the first 3 years and then annually until 10 years after the treatment. At all follow-up occasions, a physician or trained oncology nurse graded and recorded patient's highest grade of symptoms according to the international RTOG toxicity criteria [19]:

Lower urinary tract symptoms (LUTS): No (G0): 0-1 nocturia; Mild (G1): 2-3 nocturia, single dysuria/ urgency; Moderate (G2): 3-4 nocturia, sporadic dysuria/ urgency, need of medication; Severe (G3): > 4 nocturia, severe urgency, need of temporary catheterization; Permanent (G4): need of permanent catheter.

Rectal symptoms (RS): Normal (G0): 1-2 daily defecations; Mild (G1): 3-4 daily defecations, single urgency or bloody stools; Moderate (G2): > 4 daily defecations, frequent urgency or bloody stools, need of medication; Severe (G3): continuous problems and/or need of surgical treatment.

Erectile dysfunction (ED): Normal erectile function (G0); Moderate (G1): weak or not consisting of erection; Complete (G2): total loss of erectile function.

To differentiate between toxicity due to treatment and pre-existing symptoms, symptoms after the treatment were classified as toxicity if the severity (grade) was increased compared to baseline.

We, therefore, define toxicities as the occurrence of symptoms in patients without pre-existing symptoms at the same level. Toxicity was defined as acute when it occurred within 3 weeks after the end of treatment, and 
late if it occurred later (at least 6 months after the end of treatment since there was no formal follow-up between 3 weeks and 6 months).

Patients suffering from pre-existing symptoms classified with the highest grade (not considered at risk) were excluded from the analyses of that particular toxicity.

\section{Statistical analyses}

Cumulative incidences of toxicities grade $\geq 2$ were estimated by the complement of Kaplan-Meier estimator (Kaplan-Meier with reversed y-axis). Patients lost to follow-up were considered as mainly non-informative censoring, meaning they had the same risk to develop toxicities as patients still under follow-up. Death (without prior toxici-

Table 1. Baseline characteristics and dosimetric parameters. Last columns reflect relationships between parameters and toxicities grade $\geq 2$. $P$-values $<0.10$ are written out and $p$-values $>0.10$ are replaced by N.S. for "non-significant". Hyphens means that the relation was not tested due to low clinical relevance. $P$-values marked with an asterisk reflect a negative relationship

\begin{tabular}{|c|c|c|c|c|c|c|c|}
\hline Parameter (unit) & $n$ & Median (range) & $\begin{array}{l}\text { LUTS } \\
\text { acute }\end{array}$ & $\begin{array}{l}\text { LUTS } \\
\text { late }\end{array}$ & $\begin{array}{l}\text { Rectal } \\
\text { acute }\end{array}$ & $\begin{array}{l}\text { Rectal } \\
\text { late }\end{array}$ & ED \\
\hline Age (years) & 359 & $70(51-81)$ & 0.002 & N.S. & N.S. & N.S. & 0.05 \\
\hline PSA $(\mu g / l)$ & 359 & $17(1.4-253)$ & - & - & - & - & - \\
\hline Gleason & 358 & $7(5-10)$ & - & - & - & - & - \\
\hline T-stage & 359 & $2(1-3)$ & N.S. & $0.04^{*}$ & N.S. & $0.03^{*}$ & 0.07 \\
\hline Prostate volume $\left(\mathrm{cm}^{3}\right)$ & 351 & $37(16-354)$ & N.S. & N.S. & N.S. & N.S. & N.S. \\
\hline No. of pos. prostate biopsies & 349 & $5(1-8)$ & N.S. & N.S. & N.S. & $0.02^{*}$ & N.S. \\
\hline PTV $\left(\mathrm{cm}^{3}\right)$ & 331 & $33.8(15.8-99.7)$ & N.S. & 0.009 & 0.03 & 0.02 & N.S. \\
\hline$D_{90}(G y)$ & 326 & $17(9.7-253)$ & N.S. & N.S. & N.S. & N.S. & N.S. \\
\hline$V_{100}\left(\mathrm{~cm}^{3}\right)$ & 322 & 33.1 (15.5-98.9) & N.S. & 0.005 & 0.05 & 0.02 & N.S. \\
\hline$V_{200}\left(\mathrm{~cm}^{3}\right)$ & 322 & $1.6(0.1-8.5)$ & N.S. & 0.04 & N.S. & 0.02 & 0.04 \\
\hline VolU $\left(\mathrm{cm}^{3}\right)$ & 322 & $1.9(0.8-2.8)$ & N.S. & 0.02 & - & - & - \\
\hline$D_{\max } U(G y)$ & 328 & $16.0(1.6-17.3)$ & N.S. & N.S. & - & - & - \\
\hline $\mathrm{D}_{10} \mathrm{U}(\mathrm{Gy})$ & 320 & $15.5(15.1-15.8)$ & N.S. & N.S. & - & - & - \\
\hline VolRw $\left(\mathrm{cm}^{3}\right)$ & 322 & $12.8(2.9-30.5)$ & - & - & N.S. & N.S. & - \\
\hline $\mathrm{D}_{\max } \mathrm{Rw}$ (Gy) & 321 & $16.8(7.0-67.1)$ & - & - & N.S. & N.S. & - \\
\hline $\mathrm{D}_{10} \mathrm{Rw}$ (Gy) & 325 & $10.6(7.0-15.5)$ & - & - & N.S. & N.S. & - \\
\hline VolRm $\left(\mathrm{cm}^{3}\right)$ & 322 & $2.4(0.3-6.3)$ & - & - & N.S. & N.S. & - \\
\hline$D_{\max } R m$ (Gy) & 321 & $16.8(7.0-67.1)$ & - & - & N.S. & N.S. & - \\
\hline $\mathrm{D}_{10} \mathrm{Rm}$ (Gy) & 324 & $8.1(1.5-12.6)$ & - & - & N.S. & N.S. & - \\
\hline $\mathrm{D}_{0.1 \mathrm{cc}} \mathrm{Rm}$ (Gy) & 326 & $58(33-86)$ & - & - & N.S. & N.S. & - \\
\hline $\mathrm{D}_{1.0 c c} \mathrm{Rm}$ (Gy) & 326 & $45(26-76)$ & - & - & N.S. & N.S. & - \\
\hline $\mathrm{D}_{2.0 \mathrm{ccc}} \mathrm{Rm}$ (Gy) & 282 & $38(18-64)$ & - & - & N.S. & N.S. & - \\
\hline No. of HDR-BT needles & 334 & $19(14-26)$ & N.S. & N.S. & 0.0002 & N.S. & N.S. \\
\hline Lymph nodes included in EBRT target & 359 & & N.S. & N.S. & N.S. & N.S. & N.S. \\
\hline Yes & 173 & & & & & & \\
\hline No & 186 & & & & & & \\
\hline $\begin{array}{l}\text { Seminal vesicles included in EBRT } \\
\text { target }\end{array}$ & 359 & & N.S. & N.S. & N.S. & N.S. & N.S. \\
\hline Yes & 236 & & & & & & \\
\hline No & 123 & & & & & & \\
\hline ADT & 359 & & N.S. & N.S. & N.S. & N.S. & 0.003 \\
\hline Yes & 290 & & & & & & \\
\hline No & 69 & & & & & & \\
\hline
\end{tabular}

PTV - planning target volume; $D_{90}$-dose received by $90 \%$ of prostate volume; $V_{100}$ and $V_{200}$-volume of prostate receiving $100 \%$ and $200 \%$ of total dose (14.5 Gy); VolU, VolRw, and VolRm - total volume of the urethra, rectal wall, and rectal mucosa within the drawn dose-planning ultrasound image; $D_{\text {max }} U$, $D_{\text {max }} R w$, and $D_{\text {max }} R m$ - maximum dose received by the urethra, rectal wall and rectal mucosa; $D_{10} U, D_{10} R \mathrm{w}$, and $D_{10} R m$ - dose received by $10 \%$ of the most radiation-exposed urethral, rectal wall, and rectal mucosal volume; $D_{0.1 c c} R m, D_{1.0 c c} R m$, and $D_{2.0 c c} R m$-dose received by $0.1,1.0$, and $2.0 \mathrm{~cm}^{3}$ of the most radiation-exposed rectal mucosa; $E B R T$ - external beam radiotherapy; $A D T$ - androgen deprivation therapy 
ty) during follow-up was a competing event, and ignoring this might lead to a slight overestimation of a cumulative incidence, but it was considered negligible assuming that less than $10 \%$ of patients died during follow-up.

Potential predictors (which can be seen in Table 1) for toxicities grade $\geq 2$ were evaluated through bivariate analyses performed using chi-square-tests for dichotomous variables, Cochran-Armitage tests for ordinal variables, and logistic regressions for continuous variables. For urinary and rectal toxicities, independent analysis were performed for acute and late toxicities. $P$-values $<0.05$ were considered significant, indicating a potential correlation of interest. However, a large number of comparisons makes it inappropriate to draw conclusions only from $p$-values at that level.

Finally, we made multivariable analyses to evaluate more complex relationships. This could potentially result in prognostic models for predicting toxicities grade $\geq 2$. Before the multivariable analyses, all variables were controlled for collinearity. If the correlation coefficient between two variables exceeded 0.9 , one of them was removed. The multivariable analyses performed by multiple logistic regression, included all variables from the bivariate analyses (except from those removed due to collinearity). In addition, patients with incomplete data were removed from the multivariable analyses. In the bivariate analyses, acute and late rectal toxicities were analyzed separately, but the cases were too few for separate multiple logistic regressions; therefore, all rectal toxicities were analyzed together in this part.

Models were optimized by a backward stepwise procedure, minimizing AIC (Akaike information criteria). The resulting models were further trimmed by removing insignificant predictors. All predictors with $p$-values exceeding 0.05 were removed if the reduced model was not significantly $(p<0.05)$ weaker according to a likelihood ratio test. The procedure was repeated until no predictor could be removed without significantly weaken the model. All analyses were performed in R version 3.6.1.

\section{Ethical considerations}

The study protocol was approved by the Swedish Ethical Review Authority, reference number 2012/293 (2019-01719).

\section{Results}

\section{Study cohort}

In total, 359 patients were included in the study cohort. The median age was 70 (range, 51-81) years. The median follow-up was 45 (range, 2-132) months. Regarding androgen deprivation therapy (ADT), 248 patients $(69 \%)$ were treated with bicalutamide monotherapy, 27 patients $(8 \%)$ with combined ADT, and 15 patients (4\%) with GnRH agonist only.

The crude occurrence rates of symptom scoring for LUTS, RS, and ED at baseline and during follow-up of the cohort are presented in Figure 1. Since we lacked information about baseline RS for all the patients, analyses were based on an assumption that baseline status was G0 for all the patients.
Three patients were found to have distant- and five lymph node metastases directly after the end of radiotherapy. These patients remained in the analyses of toxicity rates since they received the same treatment as the others. In addition, seven patients had undergone transurethral resection of the prostate (TUR-P) prior to radiotherapy, which could theoretically affect the risk for LUTS. Since the fraction of patients with LUTS grade $\geq 2$ was not significantly different in this group compared to the others, we decided not to exclude this patient group from the analyses.

\section{Cumulative incidence of toxicities}

The estimated cumulative incidences of toxicities grade $\geq 2$ are presented in Figure 2 . The toxicity with the highest cumulative incidence was ED, followed by LUTS, whereas the incidence of RS remained low throughout the follow-up.

\section{Urinary toxicity rates over time}

Of 326 patients without pre-existing LUTS grade $\geq 2,154$ (47\%) presented with grade $\geq 2$ LUTS at any time during follow-up, 102 with acute, and 52 with late toxicities. The recovery rate of acute LUTS (based on information from the patients last contact during follow-up) was $73 \%$ ( $35 \%$ to grade $0 ; 38 \%$ to grade 1$)$, whereas the recovery rate of late LUTS was $60 \%$ ( $27 \%$ to grade $0 ; 33 \%$ to grade 1). In Figure 3, Kaplan-Meier analysis of LUTS recovery is presented. At their last follow-up, 40 (12\%) patients (of the 326 included) had a remaining grade 2 LUTS and 7 (2\%) had grade 3.

Regarding the 31 patients with pre-existing grade $\geq 2$ LUTS, 9 (29\%) were worsened at any time after the treatment. At their last follow-up, 17 patients $(55 \%)$ were improved compared to baseline, and 4 (13\%) were worsened. No grade 4 LUTS was observed at any time. Details about LUTS based on baseline status are presented in Table 2 .

\section{Rectal toxicity rates over time}

Of the 359 patients, $22(6 \%)$ presented with grade 2 RS at any time during follow-up, 6 with acute, and 16 with late toxicities. Of these 22 patients, 9 recovered to grade 1 and 9 recovered completely. 176 patients (49\%) presented with grade 1 RS at any time during follow-up, of whom 83 had acute and 93 had late toxicities. Of these 176 patients, $112(64 \%)$ recovered. No grade 3 or 4 RS was observed. Figure 3 shows Kaplan-Meier analysis of RS recovery.

\section{Erectile dysfunction rates over time}

Of 103 patients without pre-existing ED, all the patients had grade $\geq 1$ dysfunction at any time during follow-up, 84 with acute, and 19 with late toxicities. At their last follow-up, 7 patients $(7 \%)$ recovered to grade 0 , whereas 60 cases (58\%) experienced grade 1 and $36(35 \%)$ grade $2 \mathrm{ED}$. Of 114 patients with pre-existing grade $1 \mathrm{ED}$, $81(71 \%)$ had grade 2 dysfunction at any time during follow-up, 49 with acute, and 32 with late toxicities. Of the 


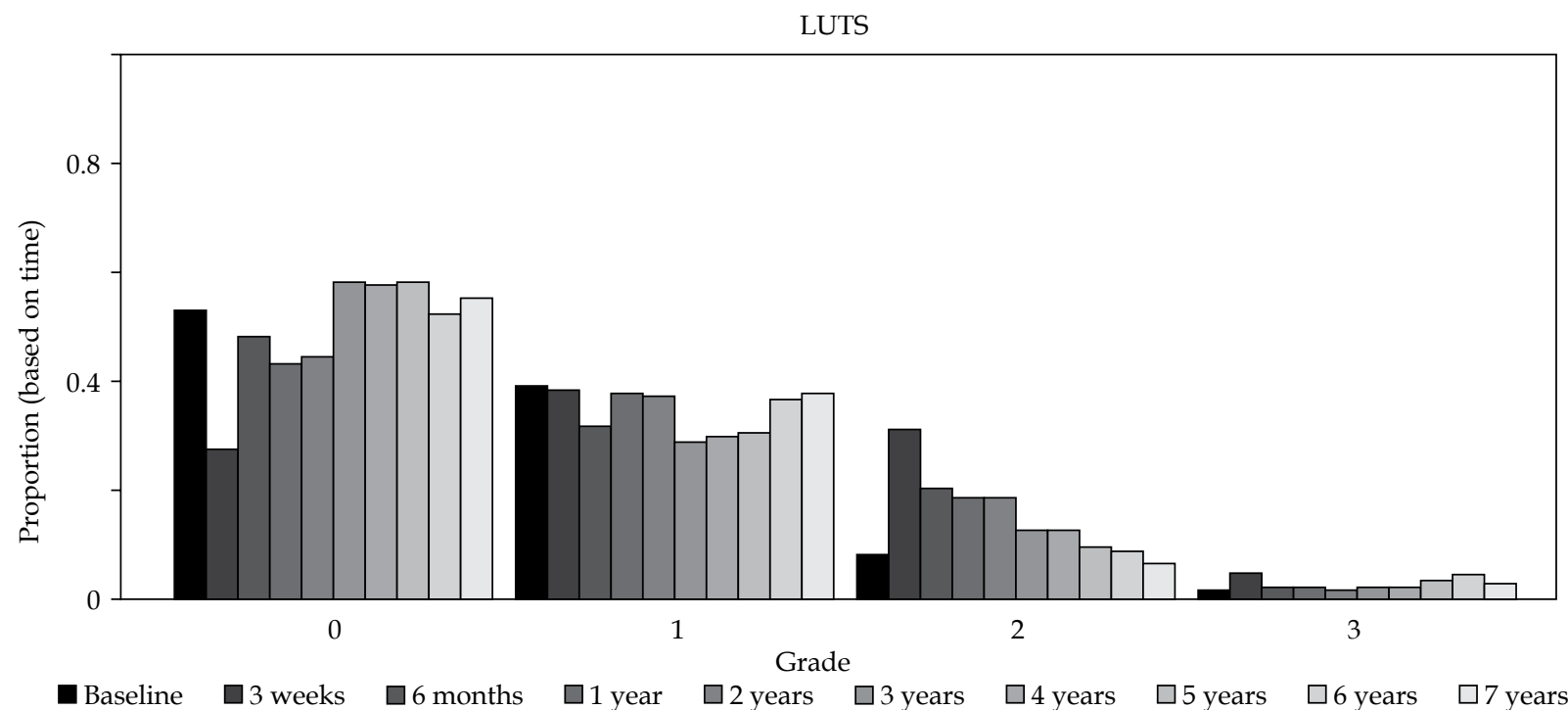

RS

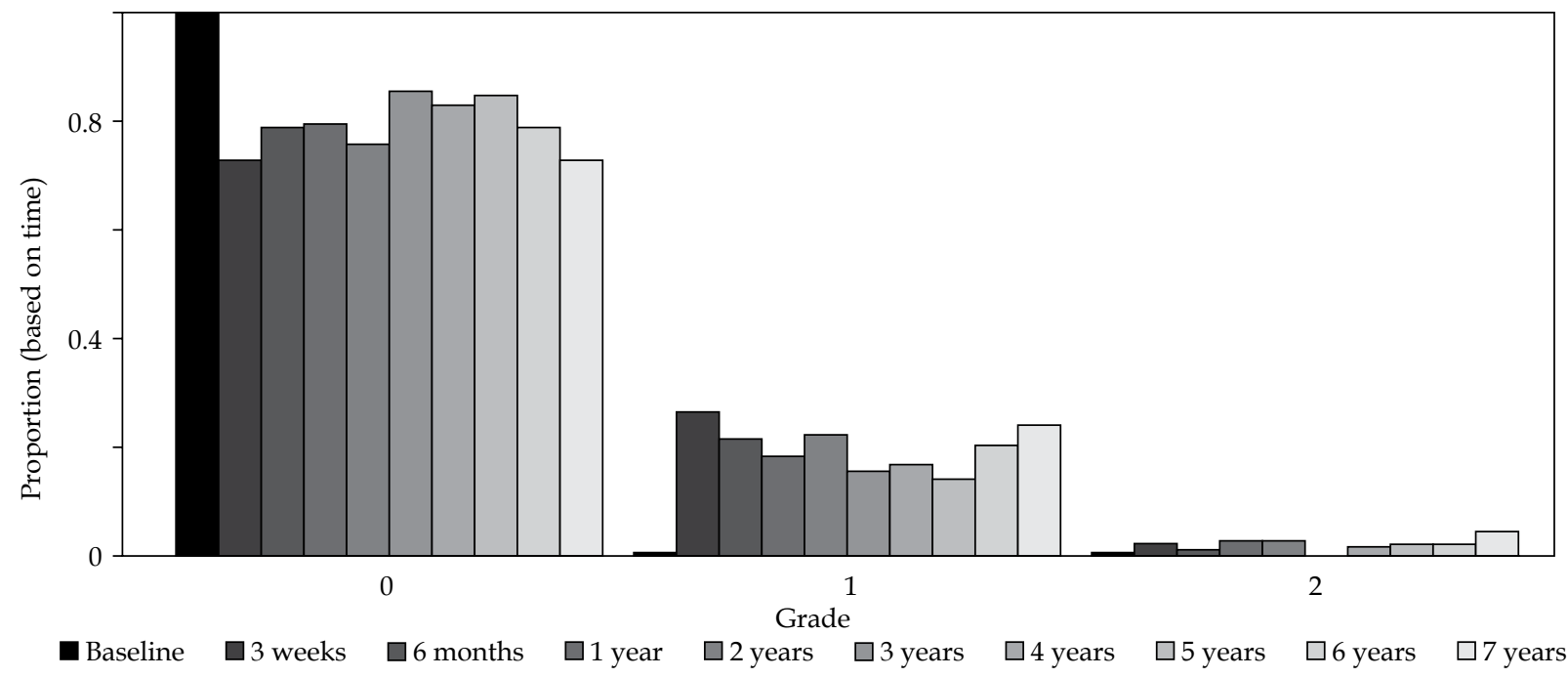

ED

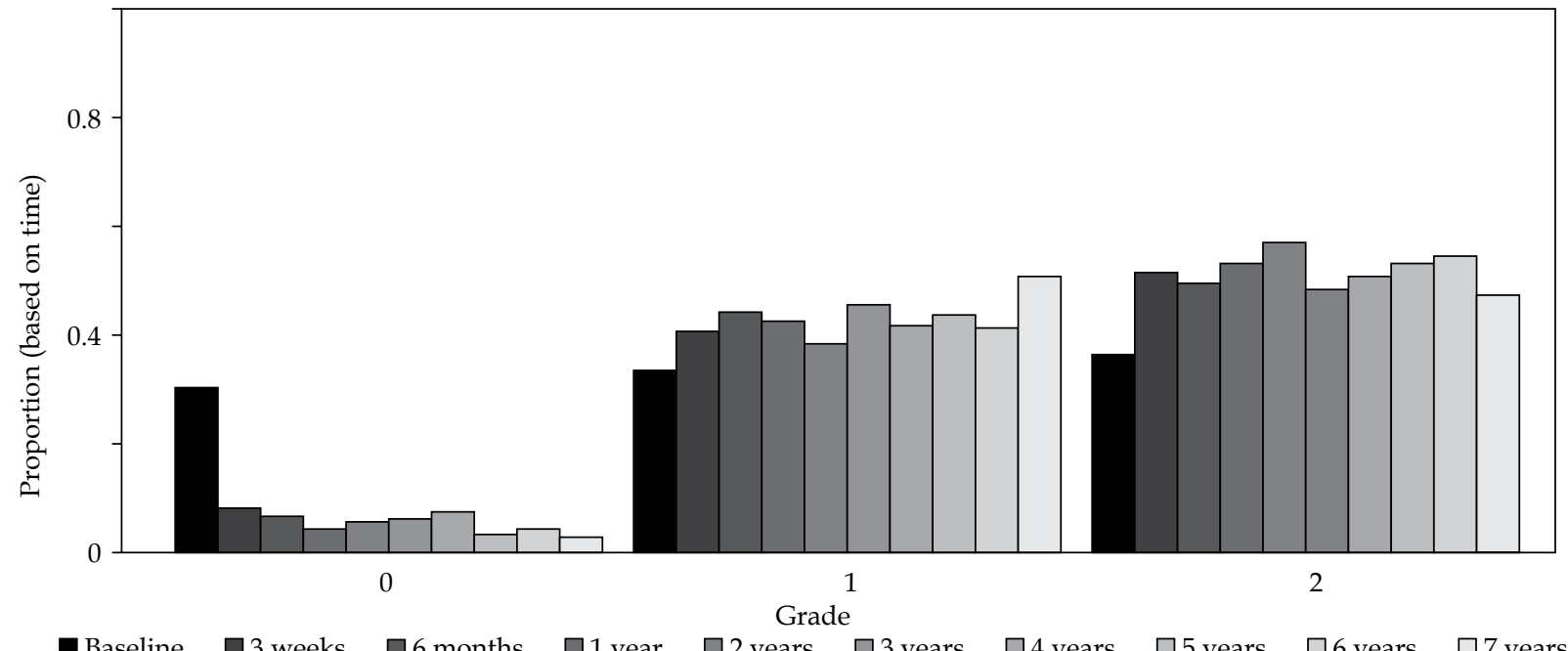

Fig. 1. Baseline and follow-up status for lower urinary tract symptoms (LUTS), rectal symptoms and erectile dysfunction. Proportion of the patients with data, $n=357$ (baseline), 356 ( 3 weeks), 328 (6 months), 306 (1 year), 262 ( 2 years), 245 ( 3 years), 145 (4 years), 133 (5 years), 75 (6 years), 51 (7 years) 


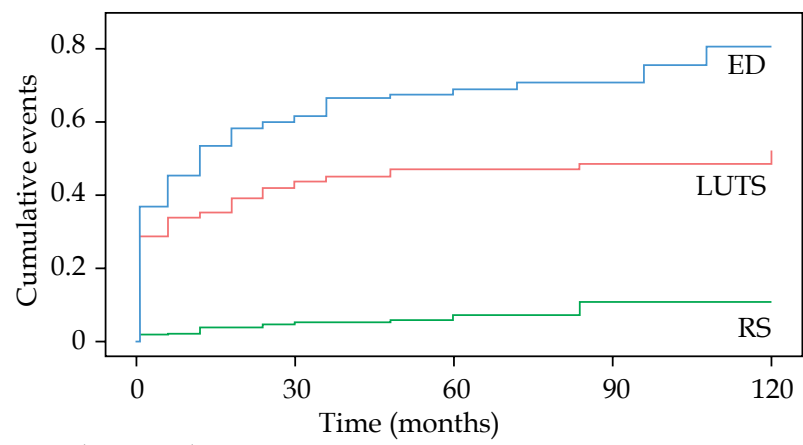

Number at risk

\begin{tabular}{lrrrrc} 
LUTS & -357 & 145 & 69 & 23 & 13 \\
RS & -359 & 264 & 130 & 33 & 17 \\
ED & -217 & 70 & 25 & 6 & 3 \\
\hline & 0 & 30 & 60 & 90 & 120 \\
& \multicolumn{3}{c}{ Time (months) }
\end{tabular}

Fig. 2. Cumulative events (complement of the Kaplan-Meier estimator). Every time a new toxicity grade $\geq 2$ arises it is counted as an event. Recovery is not taken into account. Patients with baseline ED grade 2 are excluded from the ED curve

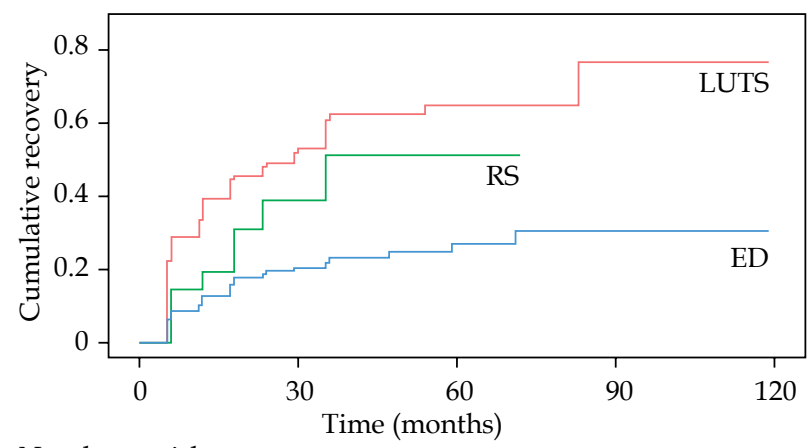

Number at risk

\begin{tabular}{lccccc} 
LUTS & -163 & 47 & 6 & 2 & 0 \\
RS & -22 & 5 & 1 & 0 & 0 \\
ED & -144 & 72 & 24 & 14 & 0 \\
\cline { 2 - 5 } & 0 & 30 & 60 & 90 & 120
\end{tabular}

Fig. 3. Cumulative recovery (complement of the Kaplan-Meier estimator). Every time a toxicity grade $\geq 2$ is improved to a lower toxicity grade it is counted as an event. Time is counted from the point where the toxicity first occurred

81 patients with grade $2 \mathrm{ED}, 28(35 \%)$ recovered to grade 1 and 52 cases $(46 \%)$ had remaining grade 2 ED at their last follow-up. Of 125 patients with pre-existing grade $2 \mathrm{ED}$, $91(73 \%)$ had remaining grade 2 dysfunction at their last follow-up, whereas $34(27 \%)$ were improved. Figure 3 demonstrates Kaplan-Meier analysis of ED recovery.

\section{Multivariable analyses}

The correlation coefficient between the independent variables exceeded 0.9 in a few cases (indicating a strong collinearity). It was 0.99 between PTV and $\mathrm{V}_{100}, 0.96$ between $\mathrm{D}_{\max } R \mathrm{~m}$ and $\mathrm{D}_{0.1 \mathrm{cc}} \mathrm{Rm}, 0.94$ between $\mathrm{D}_{0.1 \mathrm{cc}} \mathrm{Rm}$ and $\mathrm{D}_{1.0 \mathrm{cc}} \mathrm{Rm}$, and 0.93 between $\mathrm{D}_{1.0 \mathrm{cc}} \mathrm{Rm}$ and $\mathrm{D}_{2.0 \mathrm{cc}} \mathrm{Rm}$. It was
Table 2. LUTS based on baseline status. Absolute numbers with percentages in parenthesis. Patients with shorter follow-up than 6 months were excluded from the last follow-up calculations. For example, it could be seen that among patients without baseline LUTS, $44 \%$ had grade 1 LUTS after 3 weeks, and 9\% had grade 2 LUTS at their last follow-up

\begin{tabular}{lllll} 
Baseline & \multicolumn{4}{c}{ Outcomes } \\
\cline { 2 - 4 } LUS & Grade 0 & Grade 1 & Grade 2 & Grade 3 \\
\hline
\end{tabular}

Grade 0

$187(52)$

\begin{tabular}{lcccc}
3 weeks & $63(34)$ & $82(44)$ & $39(21)$ & $3(2)$ \\
\hline Highest grade & $27(14)$ & $89(48)$ & $64(34)$ & $7(4)$ \\
\hline Last follow-up & $114(61)$ & $54(29)$ & $16(9)$ & $3(2)$
\end{tabular}

Grade 1

139 (39)

\begin{tabular}{lcccc}
3 weeks & $29(21)$ & $48(35)$ & $55(40)$ & $5(4)$ \\
\hline Highest grade & $12(9)$ & $44(32)$ & $75(54)$ & $8(6)$ \\
\hline Last follow-up & $54(41)$ & $51(38)$ & $24(18)$ & $4(3)$
\end{tabular}

Grade 2

$27(8)$

\begin{tabular}{lcccc}
3 weeks & $2(8)$ & $4(15)$ & $15(58)$ & $5(19)$ \\
\hline Highest grade & $1(4)$ & $4(15)$ & $13(48)$ & $9(33)$ \\
\hline Last follow-up & $5(21)$ & $9(38)$ & $7(29)$ & $3(12)$
\end{tabular}

Grade 3

4 (1)

\begin{tabular}{lcccc}
\hline 3 weeks & $2(50)$ & $0(0)$ & $1(25)$ & $1(25)$ \\
\hline Highest grade & $0(0)$ & $1(25)$ & $2(50)$ & $1(25)$ \\
\hline Last follow-up & $2(50)$ & $0(0)$ & $1(25)$ & $1(25)$ \\
\hline Baseline total & $187(52)$ & $139(39)$ & $27(8)$ & $4(1)$ \\
\hline 3 weeks total & $96(27)$ & $134(38)$ & $110(31)$ & $14(4)$ \\
\hline Highest grade total & $40(11)$ & $138(39)$ & $154(43)$ & $25(7)$ \\
\hline Last follow-up total & $175(50)$ & $114(33)$ & $48(14)$ & $11(3)$
\end{tabular}

decided to remove PTV, $\mathrm{D}_{0.1 \mathrm{cc}} \mathrm{Rm}$, and $\mathrm{D}_{1.0 \mathrm{cc}} \mathrm{Rm}$ from the multivariable analyses.

The trimmed model for predicting acute LUTS grade $\geq 2$ was based on 304 patients and included just age $(p=0.01$, odds ratio $[\mathrm{OR}]=1.07,95 \%$ confidence interval [CI]: $1.02-1.13 \%)$.

The trimmed model for predicting late LUTS grade $\geq 2$ was based on 298 patients and included just $V_{100}$ ( $p=0.007$, OR $=1.03,95 \%$ CI: $1.01-1.05 \%)$.

The trimmed model for predicting RS grade $\geq 2$ was based on 259 patients, and included $\mathrm{V}_{100}(p=0.0009$, OR $=$ 1.10, $95 \%$ CI: $1.04-1.16 \%)$, the number of positive biopsies $(p=0.018, \mathrm{OR}=0.71,95 \% \mathrm{CI}: 0.53-0.94 \%)$, and $\mathrm{D}_{2.0 \mathrm{cc}} \mathrm{Rm}$ $(p=0.03, \mathrm{OR}=0.90,95 \%$ CI: $0.81-0.99 \%)$.

The trimmed model for predicting ED grade 2 was based on 187 patients, and included androgen deprivation therapy $(p=0.0003, \mathrm{OR}=4.07,95 \% \mathrm{CI}: 1.89-8.77 \%)$ and $\mathrm{V}_{200}(p=0.009, \mathrm{OR}=1.77,95 \% \mathrm{CI}: 1.15-2.71 \%)$. 


\section{Discussion}

In our study cohort of the 359 PC patients treated with HDR-BT combined with EBRT, we were unable to develop predictive models that could be used in clinical practice to predict treatment-related toxicities. We observed, however that both, the acute and late LUTS and RS caused by this treatment approach, were mostly mild (grade 0-1) with high recovery rates, with only few patients developed toxicities of higher grades $(\geq 2)$. For patients without pre-existing ED, all the patients presented with an impaired function after treatment, and only a few recovered completely.

Regarding toxicity rates of this treatment approach, our data are in accordance with prior cohorts using similar doses and toxicity definitions [16, 17]. The added value of our study cohort is the detailed information on the pattern of recovery after toxicity that provides some interesting insights. The recovery rates for LUTS and RS were relatively high, but with nearly half of patients suffering from any grade LUTS at the end of follow-up and one-fifth from RS. Most of the patients with remaining toxicity at the end of follow-up suffered from low-grade toxicity. Although the number of patients not recovering from LUTS or RS can be considered as low in group-level analyses, suffering from LUTS or RS due to treatment at individual level is a difficult situation that can negatively impact individual's quality of life [20]. On the contrary, the recovery pattern in patients suffering from ED was different since only few patients recovered to a normal erectile function.

In terms of identifying potential predictive factors for toxicity, we found few statistically significant correlations between certain parameters and toxicity in the bivariate analyses. However, the multivariable analyses did not provide much additional information. In summary, age seems to be a risk factor for acute LUTS, $\mathrm{V}_{100}$ (interchangeable with PTV) for late LUTS, and rectal toxicities (acute and late). For ED, usage of ADT and $V_{200}$ appears to be risk factors.

Previous studies investigating potential predictive factors for toxicity in PC patients treated with radiation therapy have shown contradictory results, with some studies suggesting a correlation between various dosimetric parameters with LUTS or RS, whereas others, including the present study, failed to confirm these correlations. Chicas-Sett et al. [16] investigated late rectal toxicity (LRT) in a cohort of 300 patients treated with a combination therapy. A significant correlation was found between $\mathrm{D}_{2.0 \mathrm{cc}}$ and LRT grade 1-3. However, no threshold dose was established because of small differences in $\mathrm{D}_{2.0 \mathrm{cc}}$ values between patients who did and did not develop toxicity; therefore, no predictive model was obtained. In another study by Kragelj et al. [17], primary objective was to assess a predictive ability of HDR-BT parameter $\mathrm{D}_{2.0 \mathrm{cc}}$ of the rectum, as the international GEC-ESTRO recommendations [20] suggest it as a predictor of LRT caused by a combination therapy. A new parameter based on the sum of mean dose from EBRT and $\mathrm{D}_{1.0 \mathrm{cc}}$ of the rectum was created, and proven to be the only statistically significant predictor of LRT.
There can be several reasons for these discrepancies, such as differences in dose constraints among treatment centers (for example, in ÖUH, the $\mathrm{D}_{10 \mathrm{Rm}}$ must be $\leq 65 \%$ of the total BT dose) as well as differences in treatment and follow-up routines. In addition, the use of different scales for grading toxicities and cut-offs (such as grade $\geq 1$ instead of $\geq 2$ ), leading to higher or lower numbers of outcome-events among studies as well as differences in baseline patients' characteristics, e.g., regarding comorbidities, could also be contributing factors to these discrepancies. Furthermore, parameters influencing statistical analyses, including sample size, number of events, and different approaches, on which parameters to include in multivariable models might also, in part, explain these discrepancies.

Due to the above-mentioned uncertainties, predictive models based on analyses from one treatment center would most likely be applicable only to patients treated and followed according to similar, if not identical, guidelines.

\section{Limitations}

The present study has several limitations that should be considered when interpreting the results. The sample size and the number of toxicity events were relatively low, thus influencing the possibility to perform statistical analyses with adequate power. In addition, the available potential predictive parameters for toxicity were fairly limited due to lack of relevant information. Specifically, dosimetric data were only retrieved from brachytherapy dose planning system, which means that dosimetric data for EBRT were not included. Furthermore, the bladder was not included in the brachytherapy dose planning; thus, no doses received by the bladder were considered in the analyses.

\section{Conclusions}

In our study cohort, we were unable to develop predictive models, which could be used in clinical practice to predict radiation-related toxicities in PC patients treated with HDR-BT and EBRT. However, the severity of toxicities due to combination therapy was in general mild or moderate and temporary with high recovery rates, whereas the incidence of severe toxicities was considerably low. Our findings are reassuring that this treatment approach as curative therapy for localized PC is well-tolerated.

\section{Acknowledgements}

We would like to thank the physicians and radiation physicists in the Oncology Department at the ÖUH, who assisted in the acquisition of all data as well as the dedicated nurses, who have followed the patients on toxicities

\section{Disclosure}

The authors report no conflict of interest. 


\section{References}

1. Bray F, Ferlay J, Soerjomataram I et al. Global cancer statistics 2018: GLOBOCAN estimates of incidence and mortality worldwide for 36 cancers in 185 countries. CA Cancer J Clin 2018; 68: 394-424.

2. Mohler JL. The 2010 NCCN clinical practice guidelines in oncology on prostate cancer. J Natl Compr Canc Netw 2010; 8: 145 .

3. Chen RC, Basak R, Meyer AM et al. Association between choice of radical prostatectomy, external beam radiotherapy, brachytherapy, or active surveillance and patient-reported quality of life among men with localized prostate cancer. JAMA 2017; 317: 1141-1150.

4. Donovan JL, Hamdy FC, Lane JA et al. Patient-reported outcomes after monitoring, surgery, or radiotherapy for prostate cancer. N Engl J Med 2016; 375: 1425-1437.

5. Hamdy FC, Donovan JL, Lane JA et al. 10-year outcomes after monitoring, surgery, or radiotherapy for localized prostate cancer. N Engl J Med 2016; 375: 1415-1424.

6. Mendez LC, Morton GC. High dose-rate brachytherapy in the treatment of prostate cancer. Transl Androl Urol 2018; 7 357-370.

7. Spratt DE, Soni PD, McLaughlin PW et al. American Brachytherapy Society Task Group Report: Combination of brachytherapy and external beam radiation for high-risk prostate cancer. Brachytherapy 2017; 16: 1-12.

8. Hoskin PJ, Rojas AM, Bownes PJ et al. Randomised trial of external beam radiotherapy alone or combined with highdose-rate brachytherapy boost for localised prostate cancer. Radiother Oncol 2012; 103: 217-222.

9. Glaser SM, Dohopolski MJ, Balasubramani GK et al. Brachytherapy boost for prostate cancer: Trends in care and survival outcomes. Brachytherapy 2017; 16: 330-341.

10. Ong WL, Evans SM, Millar JL. Under-utilisation of high-doserate brachytherapy boost in men with intermediate-high risk prostate cancer treated with external beam radiotherapy. J Med Imaging Radiat Oncol 2018; 62: 256-261.

11. Boersma LJ, van den Brink M, Bruce AM et al. Estimation of the incidence of late bladder and rectum complications after high-dose (70-78 GY) conformal radiotherapy for prostate cancer, using dose-volume histograms. Int J Radiat Oncol Biol Phys 1998; 41: 83-92.

12. Gloi AM, Buchanan R. Dosimetric assessment of prostate cancer patients through principal component analysis (PCA) J Appl Clin Med Phys 2013; 14: 3882.

13. Jackson A, Skwarchuk MW, Zelefsky MJ et al. Late rectal bleeding after conformal radiotherapy of prostate cancer. II. Volume effects and dose-volume histograms. Int J Radiat Oncol Biol Phys 2001; 49: 685-698.

14. Rancati T, Fiorino C, Gagliardi G et al. Fitting late rectal bleeding data using different NTCP models: results from an Italian multi-centric study (AIROPROS0101). Radiother Oncol 2004; 73: 21-32.

15. Söhn M, Yan D, Liang J et al. Incidence of late rectal bleeding in high-dose conformal radiotherapy of prostate cancer using equivalent uniform dose-based and dose-volume-based normal tissue complication probability models. Int J Radiat Oncol Biol Phys 2007; 67: 1066-1073.

16. Chicas-Sett R, Farga D, Perez-Calatayud MJ et al. High-doserate brachytherapy boost for prostate cancer: Analysis of dose-volume histogram parameters for predicting late rectal toxicity. Brachytherapy 2017; 16: 511-517.

17. Kragelj B, Zlatic J, Zaletel-Kragelj L. Avoidance of late rectal toxicity after high-dose-rate brachytherapy boost treatment for prostate cancer. Brachytherapy 2017; 16: 193-200.
18. Karlsson L, Thunberg P, Johansson B et al. The impact of activating source dwell positions outside the CTV on the dose to treated normal tissue volumes in TRUS guided 3D conformal interstitial HDR brachytherapy of prostate cancer. J Contemp Brachytherapy 2014; 6: 282-288.

19. Common Terminology Criteria for Adverse Events (cited 2019 Apr 27). Available from: https://www.eortc.be/ services/doc/ctc/CTCAE_4.03_2010-06-14_QuickReference_5x7.pdf

20. Hoskin PJ, Colombo A, Henry A et al. GEC/ESTRO recommendations on high dose rate afterloading brachytherapy for localised prostate cancer: an update. Radiother Oncol 2013; 107: 325-332. 